\title{
Morbidity, Mortality, and Risk Factors of Emergency Colorectal Surgery among Older Patients in the Acute Care Surgery Service
}

Chonlada Krutsri ( $\sim$ somzaisom@icloud.com )

Mahidol University Faculty of Medicine Ramathibodi Hospital

Preeda Sumpritpradit

Mahidol University Faculty of Medicine Ramathibodi Hospital

Pongsasit Singhatas

Mahidol University Faculty of Medicine Ramathibodi Hospital

Tharin Thampongsa

Mahidol University Faculty of Medicine Ramathibodi Hospital

Samart Phuwapraisirisan

Mahidol University Faculty of Medicine Ramathibodi Hospital

Goragoch Gesprasert

Mahidol University Faculty of Medicine Ramathibodi Hospital

Jakrapan Jirasiritham

Mahidol University Faculty of Medicine Ramathibodi Hospital

Pattawia Choikrua

Mahidol University Faculty of Medicine Ramathibodi Hospital

\section{Research article}

Keywords: Colorectal emergency, Colorectal surgery, Colorectal perforation, Emergency surgery, Acute Care Surgery, Older

Posted Date: June 1st, 2020

DOI: https://doi.org/10.21203/rs.3.rs-30581/v1

License: (c) (i) This work is licensed under a Creative Commons Attribution 4.0 International License. Read Full License 


\section{Abstract}

Background: Acute Care Surgery (ACS) is a rapid response system in emergency surgical conditions. Older patients have numerous factors associated with high mortality and morbidity in emergency colorectal surgery. We aimed to identify potentially preventable risk factors, to improve older patients' outcomes.

Methods: A retrospective review of patients over 60 years old undergoing emergency colorectal surgery in the ACS service from 1 August 2017 through 30 November 2019.

Results: Data of 92 patients were analyzed, average age 72.41 years. The most common diagnosis was colorectal cancer $(76,83.52 \%)$ with locations on the left $(37,41.51 \%)$, right $(35,39.33 \%)$, and rectum (17, $19.10 \%)$. Clinical presentations were obstruction without perforation $(61,67.03 \%)$, perforation $(25$, $27.17 \%)$, and ischemia $(2,2.17 \%)$. Overall mortality was $6.52 \%$. Cause of death included septic shock (3, $50 \%)$; respiratory failure $(3,50 \%)$; and pulmonary embolism $(1,16.67 \%)$. Morbidity from surgical and medical complications were $41.30 \%$ and $26.08 \%$, respectively. For all causes, operations included resection with primary anastomosis $(62,71.26 \%)$; Hartman procedure $(11,12.64 \%)$; loop colostomy (12, $13.79 \%)$; and percutaneous drainage with antibiotics (2, 2.3\%). Average operative time 159.86 minutes. In emergency colorectal surgery, preexisting heart disease, clinical perforation, and ventilator dependency increased risk of death 7.6-, 16.5-, and 0.08-fold, respectively.

Conclusion: Preexisting heart disease and clinical perforation were unmodifiable risk factors for mortality among older patients undergoing emergency colorectal surgery; ventilator dependency is potentially modifiable with advanced surgical critical care. Early, rapid, protocol-driven processes might help reduce mortality in patients with clinical presentation of perforation.

\section{Background}

The Acute Care Surgery service system is a rapid surgical response system for definite surgery under emergency surgical conditions that obviously improves patient outcome.(1)(2) Older patients who undergo colorectal surgery have numerous factors that are associated with high rates of mortality and morbidity, especially in emergency settings.(3) Emergency colorectal surgery is performed in one-fourth of all colorectal surgeries, with a $10 \%-25 \%$ mortality rate and $30 \%-50 \%$ morbidity rate in all age groups.(4) (5) The challenges to care in older people are great because of higher risks involved in surgery among patients with pre-existing comorbidities and low functional reserves, leading to higher rates of postoperative complications than in younger patients.(6) Therefore, considering the high incidence of morbidity and death after emergency colorectal surgery, it is important to determine risk factors involved in emergency surgical care, to improve patient outcomes. In this study, we aimed to identify potentially preventable or modifiable risk factors that impact mortality and morbidity if handled properly, which might improve outcomes in older patients undergoing emergency colorectal surgery. 


\section{Methods}

We collected retrospective data of patients age $\geq 60$ years who had emergency colorectal surgery in the Acute Care Surgery service of a tertiary center, Ramathibodi Hospital, from 1 August 2017 through 30 November 2019. The definition of older patients is $\geq 60$ years old in developing countries, according to the World Health Organization. Demographic data, comorbidities, hospital course, time to the operating room (OR) for source control, operative details, intraoperative fluid replacement, blood loss, operative time, and postoperative surgical and medical complications were collected from medical records. The time to OR is defined as time from diagnosis to the operating room, which is guaranteed to be within 180 minutes in the Acute Care Surgery service. Every case in the Acute Care Surgery service is first attended by a second-year general surgery resident, guaranteed to be within 30 minutes after consultation with an emergency physician and within 10 minutes by a chief resident in cases of shock or cardiac arrest. Every surgery performed or control is carried out by Acute Care Surgery staff, who are general and trauma surgeons. Postoperative Enhanced Recovery after Surgery (ERAS) protocols are applied for all patients within 3 days after surgery. Preoperative venous thromboembolism (VTE) is assessed in every case, use Caprini deep vein thrombosis (DVT) risk score; but administration of prophylaxis such as unfractionated or low-molecular-weight heparin is avoided owing to a risk of intraoperative bleeding.

Statistical analysis was conducted using Stata 14.2 software (StataCorp LLC, College Station, TX, USA). Continuous variables are summarized using mean, standard deviation, and median. Categorical variables are summarized using chi-square and presented as percentage. All descriptive statistics were stratified by mortality and morbidity. Logistic regression was used to estimate the odds ratio and $95 \%$ confidence interval (Cl) for risk factors.

This study was approved by the ethics committee of the Faculty of Medicine, Ramathibodi Hospital, Mahidol University.

\section{Results}

A total 92 older patients who had emergency colorectal surgery were included and analyzed in our study. Table 1 shows a summary of demographic data. The average patient age was 72.41 years, with 46 (50\%) men and 46 (50\%) women. Average length of stay (LOS) in the hospital was 8 days or longer if postoperative intensive care (ICU) was required. The most common comorbidities were hypertension (HT), 60 patients (65.22\%); diabetes mellitus (DM), 30 (32.61\%); dyslipidemia (DLP), 25 (27.17\%); stroke, 17 (18.48\%); and heart disease, 13 (14.13\%). Prior to surgery, 18 (19.57\%) patients were taking anticoagulants or anti-platelets. The average Caprini DVT risk score in preoperative assessment was 4 , which is compatible with moderate risk for VTE. An intermittent pneumatic compression device was used intra- and postoperatively in all patients. In a subgroup analysis between patients who survived and those who died, we found that Caprini risk score was significantly related to death, with a score of 5 in patients who died and 4 in those who survived ( $p=0.042)$. Postoperative pulmonary embolism was the cause of death in 1 patient (16.67\%). The median time to an OR was 60 minutes, which is within the guarantee. 
Twenty $(21.74 \%)$ patients had septic shock on admission, which was the cause of death in $50 \%$ of emergency colorectal surgeries in this study. Preoperative nutrition status was normal in 35 patients (49.30\%), 25 (35.21\%) had moderate malnutrition, and 11 (15.49\%) had severe malnutrition; preoperative nutrition status was not a risk factor of increased mortality: odds ratio 1.858 (95\% Cl 0.50-6.86), p=0.352 (Table 3). The overall mortality rate in older patients who had emergency colorectal surgery was $6.52 \%$. The cause of death was septic shock in 3 patients (50\%), respiratory failure in $3(50 \%)$, cardiogenic cause in $1(16.67 \%)$, pulmonary embolism in $1(16.67 \%)$, and disease process in $1(16.67 \%)$ patient. Morbidity from preexisting heart disease led to postoperative myocardial infarction in $1(1.09 \%)$ patient, congestive heart failure in $6(6.52 \%)$, and arrhythmia in $2(2.17 \%)$; these data are not shown in the table.

Table 2 presents clinical data and operative details. The most common reasons for emergency colorectal surgery were colorectal cancer in 76 (83.52\%) patients, complicated diverticulitis in 11 (12.09\%), and sigmoid volvulus in $2(2.2 \%)$ patients. The most common location of disease was the right side in 37 (41.57\%), left side in 35 (39.33\%), and rectum in 17 (20.24\%) patients. Initial presentation was obstruction in $61(67.03 \%)$, perforation in $25(27.17 \%)$, ischemia in $2(2.17 \%)$, and bleeding in $3(3.26 \%)$ patients. In subgroup analysis, we found that initial presentation of perforation was significantly associated with a high rate of death, $83.33 \%$ in patients who died versus $23.26 \%$ in those who survived $(p=0.005)$. The procedures performed included resection with primary anastomosis in $62(71.26 \%)$ patients with both right- and left-side lesions, Hartmann's operation in 11 (12.64\%), loop colostomy in 12 (13.79\%), and percutaneous drainage $2(2.3 \%)$ patients. The average preoperative lactate level was $35 \mathrm{mmol} / \mathrm{L}$, which was not significantly higher in patients who died than in those who survived: 15.9 and $2.3 \mathrm{mmol} / \mathrm{L}$, respectively $(p=0.117)$. The average operative time and blood loss was 159.86 minutes and $150 \mathrm{~mL}$, respectively. The median intraoperative fluid replacement was $1550 \mathrm{~mL}$, which was not significantly different between patients who died and those who survived $(p=0.141)$. Postoperative intensive care (ICU) was required in $26(28.89 \%)$ patients, with average LOS in the ICU of 4.5 days. LOS in the ICU was significantly higher in patients who died, 9.5 days versus 4.08 days in survivors $(p=0.020)$. The most common indication for an ICU requirement was older age in $13(14.13 \%)$ patients, septic shock in 12 (13.04\%), respiratory failure in $8(8.70 \%)$, and comorbid heart disease in $4(4.35 \%)$ patients. A total 29 $(32.22 \%)$ patients in the ICU were ventilator-dependent, which was significantly higher in patients who died $(83.33 \%)$ than in those who survived $(28.57 \%), p=0.012$. The rate of surgical complication was $41.30 \%$, which comprised surgical site infection (SSI) in $15(16.30 \%)$ patients, postoperative ileus in 8 (8.70\%), anastomosis leakage in 4 (4.35\%), and intra-abdominal collection in $4(4.35 \%)$.

Univariate analysis of risk factors is shown in Table 3, according to odds ratios. Identified risk factors in emergency colorectal surgery were comorbid heart disease, clinical perforation, and ventilator dependency, with a 7.6-, 16.5-, and 0.08 -fold increased risk of death, respectively $(p<0.05)$. The time from diagnosis to the $\mathrm{OR}$, operative time, intraoperative intravenous fluid replacement, blood loss, preoperative nutritional status, preoperative lactate level, postoperative complications, and other comorbidities were not risk factors of death. 


\section{Discussion}

Life expectancy in emergency abdominal surgery has increased, including among older patients. However, regardless of age, emergency surgery is associated with a 3- to 10-fold increased rate of operative mortality; emergency colorectal surgery has a 9-fold increased risk of death.(7)(8) A previous study found that age is not an absolute contraindication in standard colorectal surgery; however, postoperative complications, mortality, and morbidity increase with age because of low physiological reserve and pre-existing comorbidities, which affect patient outcome.(9) Acute Care Surgery is a service system for emergency general surgery in our tertiary center, Ramathibodi Hospital, with guarantees of patients being attended within 30 minutes after consultation, time to an OR within 180 minutes, and advanced surgical critical care for postoperative intensive care. Using this system, we can improve the number of non-trauma cases and patient outcomes.(1) In this study, we sought to identify risk factors of mortality and morbidity in older patients undergoing emergency colorectal surgery, with the aim to identify modifiable risk factors and improve patient outcomes.

Regarding our results, comorbid heart disease was associated with a 7.6-fold increased risk of death $(p=0.022)$, leading to postoperative myocardial infarction in $1.09 \%$, congestive heart failure in $6.52 \%$, and arrythmia in $2.17 \%$ of patients. In this study, comorbid heart disease was present in $50 \%$ of patients who died. This reflects that comorbid heart disease is an unmodifiable risk factor, and preparation of these patients in emergency settings is sometimes limited. These patients require transfer to the ICU postoperatively for close monitoring. Our results showed a postoperative ICU requirement in $28.89 \%$ of patients; by indication, rates were $4.35 \%$ for comorbid heart disease and $16.67 \%$ for postoperative death from a cardiogenic cause. A previous study among octogenarians showed a $16 \%$ probability of death from cardiovascular disease, but this was not statistically significant; neurological comorbidity had a 4fold increased risk of death.(7) Neurological comorbidities, such as stroke or even preoperative use of anticoagulants or antiplatelets, were not associated with an increased risk of death in our study.

Our service encourages an ERAS protocol for postoperative early ambulation and assessment of preoperative VTE risk using Caprini DVT risk score in every patient, to prevent adverse events of postoperative VTE. A previous report of a VTE prophylaxis benchmark for general surgery showed that VTE prophylaxis can reduce the incidence of pulmonary embolism, which can reduce mortality from $1.1 \%$ to $0.5 \%(p<0.01) .(12)$ Our study showed significant differences in Caprini risk score between patients who died and survivors, 5 and 4 , respectively $(p=0.042)$. A score of $4-5$ indicates a moderate to high risk of postoperative VTE. We therefore use intermittent pneumatic pressure in all patients intra- and postoperatively; however, we do not administer unfractionated or low-molecular-weight heparin owing to the risk of intraoperative bleeding. This prophylaxis protocol led to an incidence of death from pulmonary embolism in our study of only 1 among a total 92 cases of emergency colorectal surgery; high Caprini score was not associated with increased risk of death.

The most frequent cause of death was septic shock, with a rate of $50 \%$, mainly caused by colorectal perforation (83.33\%). Our results showed the initial clinical presentation of perforation is associated with 
a 16.5-fold increased risk of death $(p=0.013)$, especially if perforation occurred before hospitalization. With $67.03 \%$ clinical presentation of obstruction, we can prevent further perforation by not delaying surgery. In our study, the average operative time was 60 minutes. We found no further perforation with clinical obstruction, even in patients with obstruction and a competent ileocecal valve. Septic shock from perforation increases the risk of death but this may be modifiable with further development of fast-track surgical sepsis protocols to improve mortality and morbidity, which requires multidisciplinary team involvement. Calos et al. reported that septic shock was found $15.6 \%$ of emergency colorectal surgeries; in their study, this was the cause death in 5 patients but was not found to be a significant risk factor associated with death.(3)

The main cause of emergency colorectal surgery in our study population was colorectal cancer (83.52\%), the same as in previous studies.(13) Lesions were more commonly located on the right side of the colon (41.57\%) than on the left side (39.33\%). In our patients with either right-side, left-side, or rectal lesions, we performed resection with primary anastomosis to avoid colostomy formation, except in patients with high levels of contamination from perforation and those with septic shock, in whom we performed Hartmann's operation (12.64\%). The most frequently performed operation was resection with primary anastomosis $(71.26 \%)$ and anastomosis leakage (4.35\%); these rates were lower than in previous studies. As seen in Table 3, anastomosis leakage was not associated with a significantly increased risk of death.(5) This might be because anastomosis leakage can now be treated in a minimally invasive manner, such as with percutaneous drainage, bowel rest, and antibiotics. Santos et al. reported that septic shock is the leading cause of death; in their study, the most common operations were Hartmann's operation (85\%), and the mortality rate was as high as $34 \%$.(14) Compared with our results, even with increased risk of death in patients with septic shock, the overall mortality rate was only $6.52 \%$. This implies that primary anastomosis is not a contraindication in emergency colorectal surgery. Pirrera et al. reported an anastomosis leak rate of only $6.2 \%$, which confirms that older age is not a contraindication for primary anastomosis.(6) Calos et al. supported this with a low leakage rate in resection with primary anastomosis, even in emergency settings and older patients.(3) The differences in outcomes might be owing to the selection of patients for each operation; with primary anastomosis, patients with shock and high levels of contamination are avoided and Hartmann's operation is performed. Santos et al. reported that septic shock was the leading cause of death, even when the most frequent surgery was Hartmann's operation (85\%).(14) This suggests that resection with primary anastomosis is not a contraindication in emergency settings, with an anastomosis leak rate of only $4.5 \%$ and no significantly increased risk of death. Proper patient selection for each type of operation is important.

The morbidity rate from surgical complications was high at $41.30 \%$, mainly owing to minor complications such as SSI (16.30\%). Because the average time to the OR for emergency colorectal surgery was 60 minutes in our study, the time of source control in perforation was not delayed, which represents one factor that facilitated good outcomes. Patients with older ages required postoperative intensive care at a rate of $14.13 \%$. The reasons for an intensive care requirement in our older patients was owing to septic shock and respiratory failure, leading to postoperative ventilator dependency. Morbidity from ventilator dependency in older patients was high (83.33\%); in patients who died postoperatively, ventilator 
dependency had an 0.08 -fold increased risk of death $(\mathrm{p}<0.05)$. Postoperative advanced surgical critical care is important for preventing death and promoting early extubation. Preoperative nutrition status was not a significant risk factor of death, as in a previous report.(3) Average intraoperative fluid replacement was $1550 \mathrm{~mL}$, which was also not a risk factor of death; however, this may cause postoperative volume overload in patients with low cardiac functional reserve.

\section{Conclusion}

In our study, most emergency colorectal surgeries resulted from complications of colorectal cancer; older age was not a contraindication for resection with primary anastomosis. An early approach in these patients, from diagnosis to surgery, will facilitate a good patient outcome. Clinical perforation leads to sepsis and septic shock in older patients, which was the cause of death in our study; this may be modifiable to improve mortality by developing an early, rapid, protocol-driven surgical sepsis fast-track process. The nonmodifiable risk factor of comorbid heart disease might be improved by postoperative advanced critical care for close monitoring. Ventilator dependency is potentially modifiable with postoperative advanced surgical critical care. Preoperative nutrition status, other comorbidities, preoperative anticoagulant use, location of disease, operative time, intraoperative fluid replacement, and blood loss were not risk factors of increased mortality.

\section{Declarations}

Ethics approval and consent to participate: The ethics was approved and permitted by Ethical committee of Mahidol University

Consent for publication: Not applicable.

Availability of data and materials:

https://docs.google.com/spreadsheets/d/1shFlz5H5KpUJfhfnXquttQFsd9NQ3vmMYJruqBQ0068/edit

Competing interests: The authors declare that they have no competing interests.

Funding: no funding supported from any payers

\section{Authors's contributions}

Chonlada Krutsri has made substantial contributions to the conception, design of the work, interpretation of data, has drafted the work, and revised it. Preeda Sumritpradit has made substantial contributions to the conception, interpretation of data, and substantively revised it. Pongsasit Singhatas, Tharin Thampongsa, Samart Phuwapraisirisan, Goragoch Gesprasert, and Jakrapan Jirasiritham have made the acquisition, interpretation of data, and substantively revised it. Pattawia Choikrua has made an analysis, and interpretation of data. All authors read and approved the final manuscript

\section{Acknowledgment}


We thank Edanz Group (www.edanzediting.com/ac) for editing a draft of this manuscript and Miss Ticomporn Luangwattanawilai for references arrangement.

\section{References}

1. Krutsri $C$, Thampongsa $T$, Sumritpradit $P$, Singhatat $P(2018)$ Impact of an acute care surgery service on timeliness of care at Ramathibodi Hospital. J Med Assoc Thai 101:195-201.

2. Schuster KM, McGillicuddy EA, Maung AA, Kaplan LJ, Davis KA (2011) Can acute care surgeons perform emergency colorectal procedures with good outcomes? J Trauma 71:94-100; discussion -1.

3. Menegozzo CAM, Teixeira-Júnior F, Couto-Netto SDd, Martins-Júnior O, Bernini CdO, Utiyama EM (2019) Outcomes of elderly patients undergoing emergency surgery for complicated colorectal cancer: A retrospective cohort study. Clinics (Sao Paulo) 74:e1074-e.

4. Alves A, Panis Y, Mathieu P, Mantion G, Kwiatkowski F, Slim K (2005) Postoperative mortality and morbidity in French patients undergoing colorectal surgery: results of a prospective multicenter study. Arch Surg 140:278-283, discussion 84.

5. Skala K, Gervaz P, Buchs N, Inan I, Secic M, Mugnier-Konrad B, et al (2009) Risk factors for mortalitymorbidity after emergency-urgent colorectal surgery. Int $\mathrm{J}$ Colorectal Dis 24:311-316.

6. Pirrera B, Vaccari S, Cuicchi D, Lecce F, Raffele ED, Via BD, et al (2016) Impact of octogenarians on surgical outcome in colorectal cancer. Int J Surg 35:28-33.

7. Modini C, Romagnoli F, De Milito R, Romeo V, Petroni R, La Torre F, et al (2012) Octogenarians: an increasing challenge for acute care and colorectal surgeons. An outcomes analysis of emergency colorectal surgery in the elderly. Colorectal Dis 14:e312-e318.

8. Ozturk E, Yilmazlar T. Factors affecting the mortality risk in elderly patients undergoing surgery (2007) ANZ J Surg 77:156-159.

9. Basdanis G, Papadopoulos VN, Michalopoulos A, Fahantidis E, Apostolidis S, Berovalis P, et al (2004) Colorectal cancer in patients over 70 years of age: determinants of outcome. Tech Coloproctol 8 Suppl 1:s112-s115.

10. Basili G, Lorenzetti L, Biondi G, Preziuso E, Angrisano C, Carnesecchi P, et al (2008) Colorectal cancer in the elderly. Is there a role for safe and curative surgery? ANZ J Surg 78:466-470.

11. Balachandran R, Jensen KK, Burcharth J, Ekeloef S, Schack AE, Gogenur I (2020) Incidence of venous thromboembolism following major emergency abdominal surgery. World J Surg 44:704-710.

12. Golemi I, Salazar Adum JP, Tafur A, Caprini J (2019) Venous thromboembolism prophylaxis using the Caprini score. Dis Mon 65:249-298.

13. McGillicuddy EA, Schuster KM, Davis KA, Longo WE (2009) Factors predicting morbidity and mortality in emergency colorectal procedures in elderly patients. Arch Surg 144:1157-1162.

14. Santos ACd, Martins LLT, Brasil AMS, Pinto SA, Neto SG, Oliveira ECd (2014) Emergency surgery for complicated colorectal cancer in central Brazil. J Coloproctol (Rio J) 34:104-2. 


\section{Tables}


Table 1 Characteristics of older patients undergoing emergency colorectal surgery

\begin{tabular}{|c|c|c|c|c|c|}
\hline & Died & Survived & Total & \multirow{2}{*}{$\begin{array}{l}\mathrm{p}- \\
\text { value }\end{array}$} \\
\hline & & $\mathrm{N}=6$ & $N=86$ & $\mathrm{~N}=92$ & \\
\hline \multirow[t]{2}{*}{ Sex } & Male & $1(16.67)$ & $45(52.33)$ & $46(50)$ & 0.203 \\
\hline & Female & $5(83.33)$ & $41(47.67)$ & $46(50)$ & \\
\hline \multicolumn{2}{|l|}{$\begin{array}{l}\text { Age }(y) \text {, } \\
\text { mean (standard deviation) }\end{array}$} & $\begin{array}{l}83.5 \\
(10.46)\end{array}$ & $\begin{array}{l}71.63 \\
(10.42)\end{array}$ & $\begin{array}{l}72.41 \\
(10.78)\end{array}$ & 0.008 \\
\hline \multicolumn{2}{|c|}{ Total length of stay (days), median (P25, P75) } & $\begin{array}{l}13.5(6 \\
25)\end{array}$ & $8(5,17)$ & $8(6,17)$ & 0.295 \\
\hline \multicolumn{2}{|l|}{ Time to operating room (mins) } & $\begin{array}{l}80 \\
(60,120)\end{array}$ & $\begin{array}{l}60 \\
(60,120)\end{array}$ & $\begin{array}{l}60 \\
(60,120)\end{array}$ & 0.907 \\
\hline \multirow[t]{7}{*}{ Comorbidities, n (\%) } & $\begin{array}{l}\text { Arterial } \\
\text { hypertension }\end{array}$ & $4(66.67)$ & $56(65.12)$ & $60(65.22)$ & 0.999 \\
\hline & Diabetes mellitus & $2(33.33)$ & $28(32.56)$ & $30(32.61)$ & 0.999 \\
\hline & Dyslipidemia & $3(50)$ & $22(25.58)$ & $25(27.17)$ & 0.339 \\
\hline & Stroke & $2(33.33)$ & $15(17.44)$ & $17(18.48)$ & 0.306 \\
\hline & Heart disease & $3(50)$ & $10(11.63)$ & $13(14.13)$ & 0.035 \\
\hline & $\begin{array}{l}\text { Renal } \\
\text { insufficiency }\end{array}$ & $2(33.33)$ & $13(15.12)$ & $15(16.30)$ & 0.252 \\
\hline & Other disease* & $2(33.33)$ & $15(17.44)$ & $17(18.48)$ & \\
\hline \multicolumn{2}{|c|}{ Anticoagulants/antiplatelets, n (\%) } & $2(33.33)$ & $16(18.60)$ & $18(19.57)$ & 0.334 \\
\hline \multicolumn{2}{|c|}{$\begin{array}{l}\text { Caprini deep vein thrombosis risk score, } \\
\text { median (P25, P75) }\end{array}$} & $5(5,6)$ & $4(4,5)$ & $4(4,5)$ & 0.042 \\
\hline \multicolumn{2}{|l|}{ Sepsis/septic shock, n (\%) } & $3(50)$ & $17(19.77)$ & $20(21.74)$ & 0.114 \\
\hline \multirow{3}{*}{$\begin{array}{l}\text { Preoperative nutrition status, } \\
\text { n (\%) }\end{array}$} & Normal & $2(50)$ & $33(49.25)$ & $35(49.3)$ & 0.100 \\
\hline & $\begin{array}{l}\text { Moderate } \\
\text { malnutrition }\end{array}$ & 0 & $25(37.31)$ & $25(35.21)$ & \\
\hline & $\begin{array}{l}\text { Severe } \\
\text { malnutrition }\end{array}$ & $2(50)$ & $9(13.43)$ & $11(15.49)$ & \\
\hline \multicolumn{2}{|l|}{ Overall mortality (\%) } & & & $6.52 \%$ & \\
\hline \multirow[t]{2}{*}{ Cause of death, $n(\%)$} & Septic shock & $3(50)$ & & & \\
\hline & Respiratory failure & $3(50)$ & & & \\
\hline
\end{tabular}


Table 1 Characteristics of older patients undergoing emergency colorectal surgery

$\begin{array}{ll}\text { Cardiogenic cause } & 1(16.67) \\ \begin{array}{l}\text { Pulmonary } \\ \text { embolism }\end{array} & 1(16.67) \\ \text { Disease process } & 1(16.67)\end{array}$


Table 2 Surgical disease and operative details of emergency colorectal surgery

\begin{tabular}{|c|c|c|c|c|c|}
\hline & \multirow{2}{*}{$\begin{array}{l}\text { Death } \\
N=6\end{array}$} & \multirow{2}{*}{$\begin{array}{l}\text { Alive } \\
\mathrm{N}=82\end{array}$} & \multirow{2}{*}{$\begin{array}{l}\text { Total } \\
\mathrm{N}=92\end{array}$} & \multirow{2}{*}{$\begin{array}{l}\mathrm{p}- \\
\text { value }\end{array}$} \\
\hline & & & & & \\
\hline \multirow{5}{*}{$\begin{array}{l}\text { Cause of disease, } \\
\mathrm{n}(\%)\end{array}$} & Colorectal cancer & $4(66.67)$ & $\begin{array}{l}72 \\
(84.71)\end{array}$ & $\begin{array}{l}76 \\
(83.52)\end{array}$ & 0.367 \\
\hline & Complicated diverticulitis & $2(33.33)$ & $9(10.59)$ & $\begin{array}{l}11 \\
(12.09)\end{array}$ & \\
\hline & Sigmoid volvulus & 0 & $2(2.35)$ & $2(2.2)$ & \\
\hline & Rectal ischemia & 0 & $1(1.18)$ & $1(1.1)$ & \\
\hline & Metastasis & 0 & $1(1.18)$ & $1(1.1)$ & \\
\hline Location of disease, & Right side & $1(20)$ & $\begin{array}{l}36 \\
(42.86)\end{array}$ & $\begin{array}{l}37 \\
(41.57)\end{array}$ & 0.207 \\
\hline \multirow[t]{2}{*}{$n(\%)$} & Left side & $4(80)$ & $31(36.9)$ & $\begin{array}{l}35 \\
(39.33)\end{array}$ & \\
\hline & Rectum & 0 & $\begin{array}{l}17 \\
(20.24)\end{array}$ & $\begin{array}{l}17 \\
(20.24)\end{array}$ & \\
\hline Initial presentation, & Obstruction & 0 & $\begin{array}{l}61 \\
(71.76)\end{array}$ & $\begin{array}{l}61 \\
(67.03)\end{array}$ & 0.001 \\
\hline \multirow[t]{3}{*}{$n(\%)$} & Perforation & $5(83.33)$ & $\begin{array}{l}20 \\
(23.26)\end{array}$ & $\begin{array}{l}25 \\
(27.17)\end{array}$ & 0.005 \\
\hline & Ischemia & $1(16.67)$ & $1(1.16)$ & $2(2.17)$ & 0.127 \\
\hline & Bleeding & 0 & $3(3.49)$ & $3(3.26)$ & 0.999 \\
\hline Procedure, & $\begin{array}{l}\text { Resection with primary } \\
\text { anastomosis }\end{array}$ & $4(100)$ & $\begin{array}{l}58 \\
(69.88)\end{array}$ & $\begin{array}{l}62 \\
(71.26)\end{array}$ & 0.999 \\
\hline \multirow[t]{3}{*}{$n(\%)$} & Hartmann's operation & 0 & $\begin{array}{l}11 \\
(13.25)\end{array}$ & $\begin{array}{l}11 \\
(12.64)\end{array}$ & \\
\hline & Loop colostomy & 0 & $\begin{array}{l}12 \\
(14.46)\end{array}$ & $\begin{array}{l}12 \\
(13.79)\end{array}$ & \\
\hline & $\begin{array}{l}\text { Percutaneous drainage } \\
\text { and antibiotics }\end{array}$ & 0 & $2(2.41)$ & $2(2.3)$ & \\
\hline \multicolumn{2}{|c|}{$\begin{array}{l}\text { Postoperative intensive care (ICU) requirement, } \\
\text { n (\%) }\end{array}$} & $2(33.33)$ & $\begin{array}{l}24 \\
(28.57)\end{array}$ & $\begin{array}{l}26 \\
(28.89)\end{array}$ & 0.999 \\
\hline \multicolumn{2}{|c|}{$\begin{array}{l}\text { Length of stay in ICU (days), } \\
\text { mean (standard deviation, SD) }\end{array}$} & $9.5(6.36)$ & $\begin{array}{l}4.08 \\
(2.73)\end{array}$ & $4.5(3.26)$ & 0.020 \\
\hline
\end{tabular}


Table 2 Surgical disease and operative details of emergency colorectal surgery

\begin{tabular}{|c|c|c|c|c|c|}
\hline Indication for ICU, & Older age & 0 & $\begin{array}{l}13 \\
(15.12)\end{array}$ & $\begin{array}{l}13 \\
(14.13)\end{array}$ & 0.589 \\
\hline \multirow[t]{3}{*}{$n(\%)$} & Septic shock & $2(33.33)$ & $\begin{array}{l}10 \\
(11.63)\end{array}$ & $\begin{array}{l}12 \\
(13.04)\end{array}$ & 0.174 \\
\hline & Respiratory failure & $1(16.67)$ & $7(8.14)$ & $8(8.70)$ & 0.430 \\
\hline & Comorbid heart disease & 0 & $4(4.65)$ & $4(4.35)$ & 0.999 \\
\hline \multicolumn{2}{|c|}{ Postoperative ventilator dependency, n (\%) } & $5(83.33)$ & $\begin{array}{l}24 \\
(28.57)\end{array}$ & $\begin{array}{l}29 \\
(32.22)\end{array}$ & 0.012 \\
\hline \multicolumn{2}{|c|}{ Operative time (min), mean (SD) } & $\begin{array}{l}134.16 \\
(68.51)\end{array}$ & $\begin{array}{l}161.76 \\
(71.81)\end{array}$ & $\begin{array}{l}159.86 \\
(71.55)\end{array}$ & 0.365 \\
\hline \multirow{2}{*}{\multicolumn{2}{|c|}{$\begin{array}{l}\text { Intraoperative fluid replacement }(\mathrm{mL}) \text {, } \\
\text { median }(\mathrm{P} 25, \mathrm{P} 75)\end{array}$}} & $\begin{array}{l}925(550, \\
1700)\end{array}$ & 1600 & 1550 & 0.141 \\
\hline & & & $\begin{array}{l}(1000) \\
2400)\end{array}$ & $\begin{array}{l}(1000) \\
2400)\end{array}$ & \\
\hline \multicolumn{2}{|c|}{ Intraoperative blood loss (mL), median (P25, P75) } & $\begin{array}{l}75(50 \\
300)\end{array}$ & $\begin{array}{l}150(50 \\
300)\end{array}$ & $\begin{array}{l}150(50 \\
300)\end{array}$ & 0.429 \\
\hline \multicolumn{2}{|c|}{ Preoperative lactate level (mmol/mL) } & 15.9 & 2.3 & 2.35 & 0.117 \\
\hline \multicolumn{3}{|c|}{ Morbidity from surgical complication (\%) } & & $41.30 \%$ & \\
\hline \multirow{4}{*}{$\begin{array}{l}\text { Surgical } \\
\text { complications, } \\
(\%)\end{array}$} & Surgical site infection & $1(16.67)$ & $\begin{array}{l}14 \\
(16.28)\end{array}$ & $\begin{array}{l}15 \\
(16.30)\end{array}$ & 0.999 \\
\hline & Postoperative ileus & $1(16.67)$ & $7(8.14)$ & $8(8.70)$ & 0.430 \\
\hline & Anastomosis leakage & $1(16.67)$ & 3 (3.49) & $4(4.35)$ & 0.240 \\
\hline & Collection & 0 & $4(4.65)$ & $4(4.35)$ & 0.999 \\
\hline
\end{tabular}


Table 3 Univariable analysis of risk factors for morbidity and mortality

\begin{tabular}{lll}
\hline & Odds ratio $(95 \% \mathrm{Cl})$ & p-value \\
\hline Comorbid heart disease & $7.6(1.35-42.90)$ & 0.022 \\
\hline Location of disease & $1.05(0.315-3.49)$ & 0.939 \\
\hline Colorectal perforation & $16.50(0.82-149.59)$ & $\mathbf{0 . 0 1 3}$ \\
\hline Time to operating room & $1.00(0.99-1.01)$ & 0.900 \\
\hline Operative times (min) & $0.99(0.98-1.01)$ & 0.360 \\
\hline Intraoperative blood loss & $0.99(0.99-1.00)$ & 0.379 \\
\hline Intraoperative fluid replacement & $0.999(0.99-1.00)$ & 0.223 \\
\hline Preoperative nutritional status & $1.858(0.50-6.86)$ & 0.352 \\
\hline Anastomosis leakage & $5.53(0.48-63.26)$ & 0.169 \\
\hline Postoperative intensive care requirement & $0.80(0.14-4.66)$ & 0.804 \\
\hline Postoperative ventilator dependency & $0.08(0.008-0.72)$ & 0.024
\end{tabular}

